\title{
Percepción de la Calidad de Atención y Accesibilidad de los Consultorios Pediátricos Públicos en Asunción y Gran Asunción
}

\author{
MESQUITA M. ${ }^{1}$, PAVLICICH V. ${ }^{1}$, BENITEZ S. ${ }^{2}$ \\ 1. Hospital General Pediátrico "Niños de Acosta Nún" \\ 2. Centro Materno Infantil. Cátedra de Pediatría. Facultad de Medicina de la UNA.
}

\begin{abstract}
Perception of Quality of Care and Accessibility of Public Pediatric Facilities in the City of Asuncion and Greater Metropolitan Area
\end{abstract}

Introduction: It is becoming more common to analyze service in public pediatric facilities from the point of view of the children's parents. Objective: To determine the quality of care and accessibility of public pediatric facilities as perceived by patient families. Materials and Methods: The study is descriptive and observational with an analytic component. A pre-prepared questionnaire was used and filled out during an interview with parents or guardians of pediatric patients as they left the facilities of 5 hospitals in Asuncion or its metropolitan area. Results: We interviewed 208 parents or guardians. Some 58.7\% of the children were brought in due to illness, and $41 \%$ for well-child check ups. Service was described as good by $88.9 \%$ of those interviewed, while $9.1 \%$ said it was fair, and $1.9 \%$ called it poor. Reasons for describing service as good were: courteous attention $45.2 \%$, good medical care $26.4 \%$, and having received clear explanations $11 \%$. Smaller percentages mentioned having received useful information or timely attention. Of those who described the attention as fair, $63 \%$ reported receiving attention that was hurried and superficial. Of those who described it as poor, $50 \%$ did so because their children were not examined. Some $53.8 \%$ of patients arrived at the hospital by using a single bus, $23.6 \%$ used 2 buses, and 18\% arrived by walking. Smaller percentages reported using 3 buses or a private vehicle. The average cost for the visit was $9851 \pm 5645$ PYG (USD $\$ 2.48 \pm \$ 1.42$ ). Some $90 \%$ reported always having been able to get an appointment, but $58.2 \%$ said the wait was very long. There was no difference in perception according to educational level of the parents (primary education only vs. secondary education) $p=0.15$. A strong relationship was found between having received courteous attention $(p=0.001)$, the facilities of the waiting room $(p=0.012)$, a general examination of the child $(p=0.03)$, and having a play area for the children $(p=0.01)$. Conclusions: These results suggest that the parents or guardians considered courteous, personalized and compassionate attention, along with a good examination of the child and clear explanations of the child's condition, as a preponderate factor in the quality of care. Medical care is generally accessible in Asuncion and the greater metropolitan area given that the possibility exists of fees for appointments being waived in most of these hospitals.

(Key words: Perceived quality of pediatric care, accessibility of pediatric care).

Pediatr. (Asunción), 2008; 35(1): 11-7 


\section{RESUMEN}

Introducción: Analizar la calidad de la atención en los consultorios pediátricos públicos desde la percepción de los padres de los niños, es cada vez más frecuente. Objetivos: Determinar la percepción de los usuarios de la calidad de la atención en los consultorios pediátricos públicos y la accesibilidad a los mismos. Material y Método: Estudio observaciónal, descriptivo, con componente analítico; se utilizó como instrumento un cuestionario previamente elaborado que fue llenado a través de una entrevista a los padres o encargados a la salida del consultorio, en 5 hospitales de Asunción y Gran Asunción. Resultados: Se entrevistaron a 208 padres o encargados. El $58,7 \%$ de los niños fueron llevados por enfermedad y $41 \%$ para control de niño sano. El 88,9\% de los entrevistados consideró que la atención fue buena, el 9,1\% que fue regular y el 1,9\% que fue mala. Los motivos por los cuales la atención ha sido considerada como buena fueron: el $45,2 \%$ por el buen trato que recibió, el $26,4 \%$ por la buena atención médica y el $11 \%$ porque recibió explicaciones claras; en menor porcentaje por dar informaciones útiles y por la rápida atención. De los que han considerado la atención como regular, el $63 \%$ manifestó atención superficial y rápida.De los que la consideraron mala, el $50 \%$ lo hizo porque el niño no fue examinado. El 53,8\% se acercó al hospital utilizando un solo colectivo como medio de transporte, el 23,6\% utilizó 2 colectivos y el 18\% llegó caminando; en menor porcentaje han utilizado 3 colectivos y vehículo propio. El costo promedio de la consulta fue de $9851 \pm 5645$ gs; el $90 \%$ manifestó haber conseguido siempre turno para la consulta y el $58,2 \%$ consideró la espera muy larga. La percepción no fue diferente según el nivel de escolaridad de los padres (sólo estudios primarios vs estudios secundarios) $p=0,15$. Se encontró una fuerte relación de la percepción buena con: el buen trato recibido $(\mathrm{p}=0,001)$, las comodidades en la sala de espera $(p=0,012)$, el examen general del niño $(p=0,03)$ y área de juego para los mismos $(p=0,01)$. Conclusiones: Los resultados sugieren que los padres o encargados atribuyen al buen trato personal, como factor preponderante en la calidad de la atención, (personalizada y humanizada), así como al buen examen del niño y las explicaciones claras brindadas sobre el problema del mismo. La atención en general es accesible en Asunción y Gran Asunción, ya que existe la posibilidad de acceso a la exoneración de las consultas en la mayoría de estos hospitales.

(Palabras clave: Percepción de la calidad de la atención pediátrica, accesibilidad de la atención pediátrica). Pediatr. (Asunción), 2008; 35(1): 11-7

\section{ESTE TRABAJO LO PUEDE ENCONTRAR EN EXTENSO EN WWW.SciELO.ORG}

